\title{
Prevalence and mechanisms of carbapenem resistance among Klebsiella aerogenes in a tertiary hospital in China
}

\author{
Luxiang Liu \\ Zhejiang University \\ Jingjing Quan \\ Zhejiang University \\ Dongdong Zhao \\ Zhejiang University \\ Weichao Liao \\ Zhejiang University \\ Jiaojian Lv \\ Lishui Peoples' Hospital \\ Huang Zhuge \\ Lishui Peoples' Hospital \\ Feng Zhao \\ Zhejiang University \\ Yunsong Yu \\ Zhejiang University \\ Zhihui Zhou ( $\nabla$ zhouzhihui@zju.edu.cn ) \\ Zhejiang University School of Medicine Sir Run Run Shaw Hospital
}

\section{Research article}

Keywords: Klebsiella aerogenes, carbapenems, resistance

Posted Date: March 25th, 2020

DOI: https://doi.org/10.21203/rs.3.rs-19281/v1

License: (c) (i) This work is licensed under a Creative Commons Attribution 4.0 International License. Read Full License 


\section{Abstract}

Background: Klebsiella aerogenes has emerged as one of the most important nosocomial pathogens for patients in intensive care units (ICU) in recent years. This study aims to evaluated the prevalence and molecular characteristics of clinical carbapenem-resistant K. aerogenes (CRKA) isolates in a tertiary hospital in China.

Results: Twenty CRKA were identified among all the isolates, with the rate of 5.5\% (20/364). Six CRKA isolates produced KPC-2 and 1 CRKA isolate produced NDM-1. PFGE and MLST indicated that the 20 CRKA strains were clonal diversity. All the bla KPC-2 gene and bla NDM-1 gene were located on plasmids and all the plasmids with bla KPC-2 and bla NDM-1 genes could successfully transferred to EC600 or J53. Twelve of 13 CRKA strains without any carbapenemase genes were positive for efflux pump inhibition test.

Conclusion: Overall, the prevalence of CRKA in the tertiary hospital in Zhejiang Province of China is 5.5\%. Only 35\% of CRKA produce carbapenemase and efflux pumps might play an important role in the carbapenem resistance of $\mathrm{K}$. aerogenes. It is necessary to strengthen the surveillance of carbapenem resistance in the hospital to prevent the horizontal and clonal spread of CRKA.

\section{Background}

Carbapenems are the most potent and reliable $\beta$-lactam antibiotics for the treatment of serious infections caused by multidrug-resistant Enterobacteriaceae [1, 2]. However, carbapenem-resistant Enterbacteriaceae (CRE) has been gradually increased recent decades and has been the serious challenges for the public health [3]. Infections especially bloodstream infections (BSIs) with CRE are generally associated with high mortality [4]. Klebsiella aerogenes, formerly known as Enterobacter aerogenes, is a genus of a common Gram-negative, facultative anaerobic bacteria belonging to the family of Enterobacteriaceae. As opportunistic bacteria, K. aerogenes has emerged as one of the most important nosocomial pathogens for patients in intensive care units (ICU), especially for those who have mechanical ventilation [5]. Since the first report of imipenem-resistant K. aerogenes in 1990 [6], the reports about carbapenem-resistant $\mathrm{K}$. aerogenes (CRKA) have increased gradually. The prevalence of CRKA was $4.0 \%$ in France [7] and $12.5 \%$ in the USA [8], while it has reached $21 \%$ in a Chinese hospital in Shanghai [9] and $26.7 \%$ in a tertiary care hospital in Central India [10]. The mechanisms of carbapenem resistance of $\mathrm{K}$. aerogenes usually include production of carbapenemases, overproduction of $\beta$-lactamases, efflux pumps, porin deficiency, and a change in penicillin-binding proteins $[11,12]$.

In the study, we evaluated the prevalence and molecular characteristics of clinical CRKA isolates in a tertiary teaching hospital in ZheJiang province, China. Furthermore, the mechanisms of carbapenem resistance were also investigated.

\section{Results}

Prevalence of CRKA Among the $364 \mathrm{~K}$. aerogenes, 20 isolates were identified as CRKA, with the prevalent rate of 5.5\% (20/364). The resistance rates of ertapenem, meropenem and imipenem were 5.2\%, 3.0\% and 3.3\%, respectively. Most of the CRKA were resistant to ertapenem, imipenem and meropenem, except one isolate was only resistant to imipenem and four isolates were only resistant to ertapenem.

Antimicrobial susceptibility testing of CRKA Colistin, tigecycline and amikacin showed good in vitro activity against all the 20 CRKA, with susceptibility rates of $100 \%, 75 \%$ and $90 \%$, respectively. The proportions of the isolates that were resistant to cefatriaxone, cefepime, levofloxacin, piperacillin/tazobactam and cefoperazone/sulbactam were $90 \%, 70 \%, 60 \%, 65 \%, 55 \%$, respectively (Table 1) .

Characterization of carbapenemases Six of the CRKA isolates $(30 \%, 6 / 20)$ carried the gene encoding KPC-2 while one isolate $(5 \%, 1 / 20)$ carried the gene encoding NDM-1, and no other carbapenemase genes were detected. Apart from 3 CRKA that did not carry any resistance genes, other CRKA all harbored kinds of resistance genes associated with $\beta$-lactamase, quinolones, aminoglycosides or rifampicin resistance (Table 2).

PFGE and MLST seventeen distinct PFGE groups (PFGE types A-Q) were observed among the 20 CRKA isolates, among which 3 groups (type A, type $B$ and type $\mathrm{C}$ ) accounted for $10 \%(2 / 20), 10 \%(2 / 20)$ and $10 \%(2 / 20)$ of the CRKA isolates, respectively. The remaining 14 groups (D-Q) included single isolate each group. Among the 20 clinical CRKA isolates, 12 sequence types were detected, with ST4 and ST14 being the two dominant STs. It should be noted that there were 5 unknown STs marked as New in our study (Figure 1).

Transferability and location of carbapenemase genes According to the S1-PFGE and Southern blot, all the detected carbapenemase genes were located at the plasmids. The b/a $\mathrm{KPC}_{2}$ carried by strains 11907, 15423, 34366 and 34602 were located at plasmids of similar size, ranging from about $78.2 \mathrm{~kb}$ to $104.5 \mathrm{~kb}$, while $b / a_{\mathrm{KPC}-2}$ carried by strains 12517 and 15923 were located at plasmids with the same size of $104.5 \mathrm{~kb}$. And the bla $a_{\mathrm{NDM}-1}$ carried by strain

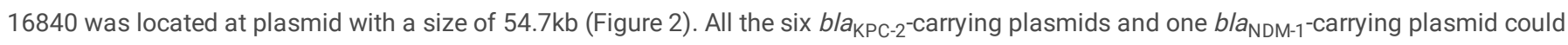
successfully transferred from $K$. aerogenes to E.coli EC600 or E. coli J53. The Seven E. coli transconjugants acquired $b / a_{\mathrm{KPC}-2}$ or bla $a_{\mathrm{NDM}-1}$ genes and exhibited significantly decreased carbapenem susceptibility. The MICs of imipenem, meropenem and ertapenem ranged from 2 to $64 \mathrm{ug} / \mathrm{mL}$ and the antimicrobial susceptibility patterns of E. coli transconjugants were similar to those of the donor (Supplemental table 2).

Detection of efflux pump activity All of the 13 CRKA that were absent of carbapenemase were performed efflux pump inhibition test. A greater than or equal to 4 folds concentration decrease in MIC for at least one carbapenem tested in combination with PABN or CCCP was shown in (53.8\%, 7/13) and 
$(92.3 \%, 12 / 13)$ of the CRKA strains, respectively. This indicated that the activation of efflux pumps might contribute to the carbapenem resistance of CRKA isolates (except the isolate 33127) (Table3).

\section{Discussion}

K. aerogenes has regularly been involved in nosocomial infections outbreaks since 1993, particularly in the Western Europe [13-16]. Since 2010, K. aerogenes is the fifth highest Enterobacteriaceae and the seventh highest Gram-negative Bacillus responsible for notorious nosocomial infections in France [17].

In the present study, twenty CRKA were identified among all isolates, with the rate of 5.5\% (20/364), slightly higher than the France (4.0\%) [7], but much lower than some other countries or regions (The USA 12.5\% [8], India 26.7\% [10]), including Shanghai (21\%) [9]. Although CRKA generally represents a low proportion of CRE compared to carbapenem-resistant K. pneumoniae, our findings demonstrated that CRKA had emerged in the clinics.

Carbapenamase production is one of the main resistance mechanisms of CRE. Carbapenemases consist of ambler class A or D serine $\beta$-lactamases and ambler class B metallo- $\beta$-lactamases (MBLs). K. pneumoniae carbapenemase (KPC, Class A) is the most common carbapenemase in carbapenemase producing Enterobacteriaceae (CPE) [3]. It was first discovered in the USA in 1996 [18], and has spread worldwide since then. Recently years, KPCproducing Enterobacteriaceae have become a public health concern worldwide, including in China [19, 20]. However, in this study, only six CRKA and one CRKA were identified as producing KPC-2 and NDM-1. Further analysis of PFGE and MLST revealed that most of the KPC-2-producing CRKA were nonclonal spread, except two CRKA (34366 and 34602), which were isolated from the same department. Several study had reported that KPC-2-producing CRKA once caused an epidemic and outbreak in hospitals of China $[9,21]$. In our study, S1-PFGE followed by Southern blot demonstrated that all the

bla $_{\mathrm{KPC}-2}$ and bla $\mathrm{NDM}_{\mathrm{N}}$ genes were all located at plasmids. Furthermore, the filter mating experiments confirmed that all the bla $\mathrm{KPC}_{2}$ and

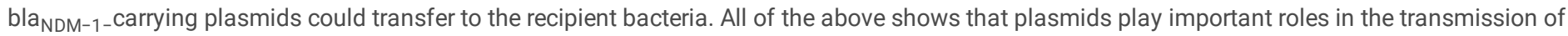
carbapenemase genes and indicate the potential threat of outbreak and prevalence of CRKA.

In addition to carbapenemases, other mechanisms such as the overexpression of efflux pumps contributing to the carbapenem resistance in $\mathrm{K}$. pneumoniae are now well documented [22]. The efflux pump inhibitors PAßN and CCCP are active against RND pumps in Gram-negative bacteria, including E. cloacae, E. coli, K. pneumoniae, P. aeruginosa, S. enteric and so on [23]. Despite of the potential toxic effect of PABN and CCCP on bacteria, concentrations of $25 \mu \mathrm{g} / \mathrm{ml}$ of PABN and $25 \mu \mathrm{g} / \mathrm{ml}$ of CCCP could not kill bacteria [23,24]. Our results showed that the MICs of CRKA isolates could decreased significantly in the presence of PABN and CCCP, indicating that efflux pumps might play an important role in the carbapenem resistance of $\mathrm{K}$. aerogenes. Moreover, there were still 1 isolates of CRKA without carbapenase and had no efflux pump inhibitor inhibition phenotype in the present study. Further research is needed to confirm the mechanisms of carbapenem resistance.

\section{Conclusion}

Overall, the prevalence of CRKA in the tertiary hospital in Zhejiang Province of China is 5.5\% during the years from 2011 to 2017 . Only $35 \%$ of CRKA produce carbapenemase and efflux pumps might play an important role in the carbapenem resistance of $\mathrm{K}$. aerogenes. bla $\mathrm{KPC}_{2}$ and bla $\mathrm{NDM}_{-1}$ are major carbapenemase genes and always transfer through plasmids. It is necessary to strengthen the surveillance of carbapenem resistance in the hospital to prevent the horizontal and clonal spread of CRKA.

\section{Methods}

Bacterial isolates All the $K$. aerogenes isolates ( $n=364$ ) were collected from 2011-2017 in a tertiary hospital in Zhejiang province, China (isolates of 2014 were lost). These isolates were non-duplicate and were collected on routine workdays without any specific exclusion criteria. Escherichia coli strain ATCC 25922 was used as control for antimicrobial susceptibility testing. In addition, E. coli EC600 (resistant to rifampicin) and E. coli J53 (resistant to Sodium azide) were used as recipients for conjugation test.

Antimicrobial susceptibility testing Antimicrobial susceptibility testing was performed using the microbroth dilution method recommended by the Clinical and Laboratory Standards Institute (CLSI) [25]. Breakpoint MICs of tigecycline and colistin were determined following the guidelines of the US Food and Drug Administration (with MICs $\leq 2 \mathrm{ug} / \mathrm{mL}$ denoting susceptibility and $\geq 8 \mathrm{ug} / \mathrm{ml}$ denoting resistance) and the EUCAST 2018 (with MICs $\leq 2 \mathrm{ug} / \mathrm{ml}$ denoting susceptibility and $\geq 4 \mathrm{ug} / \mathrm{ml}$ denoting resistance) (http://www.eucast.org/clinical_breakpoints/), respectively. And the breakpoints MICs of cefatriaxone, cefepime, levofloxacin, amikacin, ertapenem, meropenem, imipenem, piperacillin/tazobactam and cefoperazone/sulbactam were determined following criteria of the CLSI 2018 [25].

Detection of carbapenemase genes The presence of genes encoding the carbapenemases KPC, NDM, VIM, IMP, and OXA-48 were investigated in all of the CRKA isolates using the primers described in Supplemental table 1. The amplified products were observed and confirmed by agarose gel electrophoresis and the positive ones were sequenced with Sanger sequencing. The sequences were further confirmed using BLAST searches (https://blast.ncbi.nlm.nih.gov/Blast.cgi).

Pulsed field gel electrophoresis (PFGE) Clonal relationships of CRKA were analysed using PFGE, which was performed according to a previously described protocol. Briefly, the DNA fragments were separated with a CHEF-Mapper XA PFGE system (Bio-Rad, USA). Electrophoresis was performed for

Page $3 / 8$ 
22 hours at $14^{\circ} \mathrm{C}$ with pulse times ranging from 5 to $35 \mathrm{~s}$ at $6 \mathrm{~V} / \mathrm{cm}$. Salmonella strain $\mathrm{H} 9812$ was used as the molecular marker. The DNA fingerprints generated were analysed according to the criteria proposed by TENOVER et al [19].

S1-PFGE and Southern blot hybridization The location of the carbapenemase genes were determined by S1-nuclease digestion and pulsed-field gel electrophoresis (S1-PFGE) combined with Southern blotting hybridizations. Gegomic DNA of all carbapenemases-producing isolates were extracted and embedded in gold agarose gel plugs (SeaKem ${ }^{\circledR}$ Gold Agarose, Lonza, Atlanta, GA USA). The plugs were digested with S1 nuclease (TaKaRa, Dalian, China), and the DNA fragments were separated by PFGE. The plasmid was characterized by S1-PFGE, and the location of carbapenemase genes was identified by Southern hybridization with digoxigenin-labelled probe using the DIG-High Prime DNA Labeling and Detection Starter Kit II (Roche Diagnostics).

Transferability of carbapenemase genes Conjugation experiments were carried out in Luria-Bertani broth with E. coli EC600 (resistant to rifampicin) or $E$. coli J53 (resistant to Sodium azide) as the recipient as described previously [26]. E. coli transconjugants were selected on Mueller-Hinton agar containing rifampicin $(600 \mathrm{ug} / \mathrm{ml})$ or Sodium azide $(200 \mathrm{ug} / \mathrm{ml})$ and ertapenem $(2 \mathrm{ug} / \mathrm{ml})$. The colonies that grew on the selecting medium were picked up and identified using the MALDI-TOF and PCR analysis.

Whole genome sequencing The whole genomes of all the CRKA strains were extracted and sequenced by Illumina HiSeq2000 platform. Sequencing data was assembled by CLC Genomics Workbench software (Version 9.0). The multi-locus sequence type (MLST) and other resistant genes were analyzed on the website of Center of Genomics Epidemiology (http://www.genomicepidemiology.org/).

Efflux pump inhibition test In addition, to investigate the role of efflux pumps in the carbapenem resistance mechanisms, the MICs of carbapenems were determined in the presence of the efflux pump inhibitor Phe-Arg $\beta$-naphthylamide dihydrochloride (PABN:25ug/ml) [27] and carbonyl cyanide $\mathrm{m}$ chlorophenylhydrazone (CCCP:25ug/ml) [9], respectively. A 4-fold or greater reduction in the MIC values after addition of CCCP or PABN was considered as significance [28].

\section{Abbreviations}

CRKA: carbapenem-resistant Klebsiella aerogenes; CRE: carbapenem-resistant Enterbacteriaceae; BSI: bloodstream infection; ICU: intensive care units; PFGE: pulsed-field gel electrophoresis; MLST: multi-locus sequence type; MIC: minimum inhibition concentration; CLSI: Clinical and Laboratory Standards Institute; FDA: US Food and Drug Administration; PAßN: Phe-Arg $\beta$-naphthylamide dihydrochloride; CCCP: carbonyl cyanide mchlorophenylhydrazone.

\section{Declarations}

\section{Ethics approval and consent to participate}

The clinical isolates were part of the routine hospital laboratory procedure. The present study mainly focused on bacteria, but not the patients. So the ethical approval was not required.

\section{Consent for publication}

Not applicable.

\section{Availability of data and materials}

All data generated or analyzed during this study are included in this published article and its supplementary information files.

\section{Funding}

This study was funded by the National Natural Science Foundation of China (81830069) and the Key Research and Development Programme of Zhejiang (2015C03046).

\section{Competing interests}

The authors declare that they have no competing interests.

\section{Authors' contributions}

All authors participated in the conception and design of the study; conceived and drafted the manuscript: LLX, ZZH and QJJ; performed the experiments: QJJ, LWC and ZF; analyzed the data: LLX, LJJ and ZGH; revised the paper: ZZH, ZDD and YYS. All authors read and approved the final manuscript.

\section{Acknowledgements}

Not applicable. 


\section{References}

1. Vardakas KZ, Tansarli GS, Rafailidis PI, Falagas ME: Carbapenems versus alternative antibiotics for the treatment of bacteraemia due to Enterobacteriaceae producing extended-spectrum beta-lactamases: a systematic review and meta-analysis. J Antimicrob Chemother 2012 , 67(12):2793-803.

2. El-Gamal MI, Brahim I, Hisham N, Aladdin R, Mohammed H, Bahaaeldin A: Recent updates of carbapenem antibiotics. Eur J Med Chem 2017, 131:185-95

3. Potter RF, D'Souza AW, Dantas G: The rapid spread of carbapenem-resistant Enterobacteriaceae. Drug Resist Updat 2016, 29:30-46.

4. van Duin D, Kaye KS, Neuner EA, Bonomo RA: Carbapenem-resistant Enterobacteriaceae: a review of treatment and outcomes. Diagn Microbiol Infect Dis 2013, 75(2):115-20.

5. Mezzatesta ML, Gona F, Stefani S: Enterobacter cloacae complex: clinical impact and emerging antibiotic resistance. Future Microbiol 2012, 7(7):887-902.

6. Hopkins JM, Towner KJ: Enhanced resistance to cefotaxime and imipenem associated with outer membrane protein alterations in Enterobacter aerogenes. J Antimicrob Chemother 1990, 25(1):49-55.

7. Robert J, Pantel A, Merens A, Lavigne JP, Nicolas-Chanoine MH, Group ONsCRS: Incidence rates of carbapenemase-producing Enterobacteriaceae clinical isolates in France: a prospective nationwide study in 2011-12. J Antimicrob Chemother 2014, 69(10):2706-12.

8. Guh AY, Bulens SN, Mu Y, Jacob JT, Reno J, Scott J, Wilson LE, Vaeth E, Lynfield R, Shaw KM et al: Epidemiology of Carbapenem-Resistant Enterobacteriaceae in 7 US Communities, 2012-2013. JAMA 2015, 314(14):1479-87.

9. Qin X, Yang Y, Hu F, Zhu D: Hospital clonal dissemination of Enterobacter aerogenes producing carbapenemase KPC-2 in a Chinese teaching hospital. J Med Microbiol 2014, 63(Pt 2):222-8.

10. Khajuria A, Praharaj AK, Kumar M, Grover N: Carbapenem Resistance among Enterobacter Species in a Tertiary Care Hospital in Central India. Chemother Res Pract 2014, 2014:972646.

11. Codjoe FS, Donkor ES: Carbapenem Resistance: A Review. Med Sci (Basel) 2017, 6(1).

12. Suay-Garcia B, Perez-Gracia MT: Present and Future of Carbapenem-resistant Enterobacteriaceae (CRE) Infections. Antibiotics (Basel) 2019, 8(3).

13. Georghiou PR, Hamill RJ, Wright CE, Versalovic J, Koeuth T, Watson DA, Lupski JR: Molecular epidemiology of infections due to Enterobacter aerogenes: identification of hospital outbreak-associated strains by molecular techniques. Clin Infect Dis 1995, 20(1):84-94.

14. Davin-Regli A, Monnet D, Saux P, Bosi C, Charrel R, Barthelemy A, Bollet C: Molecular epidemiology of Enterobacter aerogenes acquisition: one-year prospective study in two intensive care units. J Clin Microbiol 1996, 34(6):1474-80.

15. De Gheldre Y, Maes N, Rost F, De Ryck R, Clevenbergh P, Vincent JL, Struelens MJ: Molecular epidemiology of an outbreak of multidrug-resistant Enterobacter aerogenes infections and in vivo emergence of imipenem resistance. J Clin Microbiol 1997, 35(1):152-60.

16. Jalaluddin S, Devaster JM, Scheen R, Gerard M, Butzler JP: Molecular epidemiological study of nosocomial Enterobacter aerogenes isolates in a Belgian hospital. J Clin Microbiol 1998, 36(7):1846-52.

17. Carbonne A, Arnaud I, Maugat S, Marty N, Dumartin C, Bertrand X, Bajolet O, Savey A, Fosse T, Eveillard M et al: National multidrug-resistant bacteria (MDRB) surveillance in France through the RAISIN network: a 9 year experience. J Antimicrob Chemother 2013, 68(4):954-9.

18. Yigit H, Queenan AM, Anderson GJ, Domenech-Sanchez A, Biddle JW, Steward CD, Alberti S, Bush K, Tenover FC: Novel carbapenem-hydrolyzing beta-lactamase, KPC-1, from a carbapenem-resistant strain of Klebsiella pneumoniae. Antimicrob Agents Chemother 2001, 45(4):1151-61.

19. Tenover FC, Arbeit RD, Goering RV, Mickelsen PA, Murray BE, Persing DH, Swaminathan B: Interpreting chromosomal DNA restriction patterns produced by pulsed-field gel electrophoresis: criteria for bacterial strain typing. J Clin Microbiol 1995, 33(9):2233-9.

20. Hu F, Chen S, Xu X, Guo Y, Liu Y, Zhu D, Zhang Y: Emergence of carbapenem-resistant clinical Enterobacteriaceae isolates from a teaching hospital in Shanghai, China. J Med Microbiol 2012, 61(Pt 1):132-6.

21. Hao M, Shen Z, Ye M, Hu F, Xu X, Yang Y, Wu S, Lin D, Qin X, Wang M: Outbreak Of Klebsiella pneumoniae Carbapenemase-Producing Klebsiella aerogenes Strains In A Tertiary Hospital In China. Infect Drug Resist 2019, 12:3283-90.

22. Martins A, Spengler G, Martins M, Rodrigues L, Viveiros M, Davin-Regli A, Chevalier J, Couto I, Pages JM, Amaral L: Physiological characterisation of the efflux pump system of antibiotic-susceptible and multidrug-resistant Enterobacter aerogenes. Int J Antimicrob Agents 2010, 36(4):313-8.

23. Blair JM, Piddock LJ: Structure, function and inhibition of RND efflux pumps in Gram-negative bacteria: an update. Curr Opin Microbiol 2009, 12(5):512-9.

24. Szabo D, Silveira F, Hujer AM, Bonomo RA, Hujer KM, Marsh JW, Bethel CR, Doi Y, Deeley K, Paterson DL: Outer membrane protein changes and efflux pump expression together may confer resistance to ertapenem in Enterobacter cloacae. Antimicrob Agents Chemother 2006, 50(8):2833-5.

25. CLSI.M100 Performance Standards for Antimicrobial Susceptibility Testing. Wayne, PA:Clinical and Laboratory Standards Institute. 2018.

26. Zhang R, Wang XD, Cai JC, Zhou HW, Lv HX, Hu QF, Chen GX: Outbreak of Klebsiella pneumoniae carbapenemase 2-producing K. pneumoniae with high qnr prevalence in a Chinese hospital. J Med Microbiol 2011, 60(Pt 7):977-82.

27. Yang FC, Yan JJ, Hung KH, Wu JJ: Characterization of ertapenem-resistant Enterobacter cloacae in a Taiwanese university hospital. J Clin Microbiol 2012, 50(2):223-6. 
28. Schumacher A, Steinke P, Bohnert JA, Akova M, Jonas D, Kern WV: Effect of 1-(1-naphthylmethyl)-piperazine, a novel putative efflux pump inhibitor, on antimicrobial drug susceptibility in clinical isolates of Enterobacteriaceae other than Escherichia coli. J Antimicrob Chemother 2006, 57(2):344-8.

\section{Tables}

Table 1. In-vitro activities of antimicrobial agents against CRKA isolates.

ntibiotics $\mathrm{MIC}_{50} \mathrm{MIC}_{90} \mathrm{MIC}$ range $\mathrm{S}(\%) \mathrm{I}(\%) \mathrm{R}(\%)$

$\mu \mathrm{g} / \mathrm{mL} \mu \mathrm{g} / \mathrm{mL} \quad \mu \mathrm{g} / \mathrm{mL}$

\begin{tabular}{ccccccc}
\hline TX & 64 & $\square 256$ & $0.03-\square 256$ & 10 & 0 & 90 \\
FEP & 32 & $\square 256$ & $0.008-256$ & 25 & 5 & 70 \\
LEV & 2 & 16 & $0.06-64$ & 5 & 35 & 60 \\
ETP & 16 & 32 & $0.25-\llbracket 256$ & 5 & 0 & 95 \\
MEM & 4 & 16 & $0.125-128$ & 35 & 10 & 55 \\
IPM & 4 & 64 & $0.5-64$ & 30 & 10 & 60 \\
PTC & 256 & $\square 512$ & $2-512$ & 20 & 15 & 65 \\
CPS & 32 & $\square 128$ & $0.25-\square 128$ & 35 & 10 & 55 \\
AMK & 2 & 16 & $1-256$ & 90 & 0 & 10 \\
CST & 0.06 & 0.125 & $0.015-2$ & 100 & 0 & 0 \\
TGC & 1 & 8 & $0.125-16$ & 75 & 10 & 15
\end{tabular}

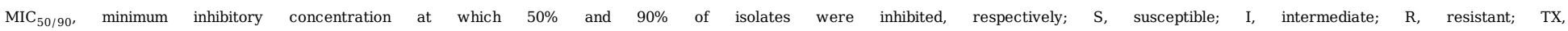

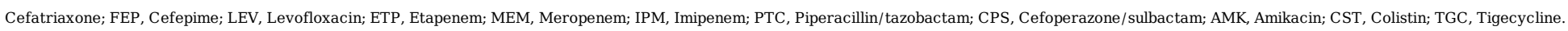

Table 2. Resistance genes carried by CRKA.

\begin{tabular}{|c|c|c|c|c|}
\hline Isolates & $\beta$-Lactamase & Quinolones & Aminoglycosides & Rifampicin \\
\hline 11914 & - & - & - & - \\
\hline 12173 & bla $_{\mathrm{SHV}-120}$ & qnrS1 & - & - \\
\hline 12517 & $b l a_{\mathrm{KPC}-2 \square} b l a_{\mathrm{CTX}-\mathrm{M}-14}$ & $q n r S 1 \square a a c\left(6^{\prime}\right)-I b-c r$ & $\operatorname{aac}\left(6^{\prime}\right)-I b 3$ & $A R R-3$ \\
\hline 12888 & - & qnrS1 & - & - \\
\hline 13274 & - & qnrS1 & $\operatorname{aph}\left(3^{\prime \prime}\right)-\operatorname{Ib} \rrbracket a p h(6)-I d \square \operatorname{aad} A 2$ & - \\
\hline 13322 & - & - & - & - \\
\hline 13469 & bla $_{\text {SHV-120 }}$ & $q n r S 1$ & - & - \\
\hline \multirow[t]{2}{*}{13865} & $b l a_{\text {TEM-1B }} b l a_{\text {OXA-1 }}$ & $a a c\left(6^{\prime}\right)-I b-c r$ & aac(3)-IIa $\amalg a p h\left(3^{\prime \prime}\right)-I b$ & - \\
\hline & $b_{\text {CTX-M-15 }}$ & & $\operatorname{aph}(6)-I d$ & \\
\hline 15322 & - & qnrS1 & - & - \\
\hline 15390 & - & - & - & - \\
\hline \multirow[t]{2}{*}{15423} & $b l a_{\mathrm{KPC}-2 \rrbracket} b l a_{\mathrm{TEM}-1 \mathrm{C}}$ & $q n r S 1$ & aadA1 & $A R R-2$ \\
\hline & $b l a_{\mathrm{OXA}-10}$ & & & \\
\hline 15923 & $b l a_{\text {KPC-2凸}} b l a_{\text {CTX-M-14 }}$ & qnrS1 & & \\
\hline 16010 & $b_{\text {CTX-M-27 }}$ & aac $\left(6^{\prime}\right)-I b-c r \rrbracket q n r B 52$ & aadA16 & $A R R-3$ \\
\hline 16840 & $b l a_{\mathrm{NDM}-1 \square} b l a_{\mathrm{SHV}-12}$ & - & - & - \\
\hline 33127 & - & $q n r S 1$ & - & - \\
\hline 34102 & - & - & - & - \\
\hline 34366 & $b l a_{\mathrm{KPC}-2 \square} b l a_{\mathrm{TEM}-1 \mathrm{~B}}$ & $q n r S 1$ & - & - \\
\hline 34602 & $b l a_{\mathrm{KPC}-2}$ & $q n r S 1$ & - & - \\
\hline \multirow[t]{2}{*}{11907} & $b l a_{\mathrm{KPC}-2 \rrbracket} b l a_{\mathrm{CTX}-\mathrm{M}-14}$ & $q n r S 1$ & aac $\left(6^{\prime}\right)-I b-H a n g z h o u$ & - \\
\hline & $b l a_{\text {TEM-1B }}$ & $a a c\left(6^{\prime}\right)-I b-c r$ & - & - \\
\hline 131029 & $b l a_{\text {TEM-1B }}$ & $q n r S 1$ & - & - \\
\hline
\end{tabular}

Table 3. MICs of carbapenems with or without PABN or CCCP for 13 CRKA isolates 


\begin{tabular}{|c|c|c|c|c|c|c|}
\hline \multirow[t]{2}{*}{ Isolates } & \multicolumn{6}{|c|}{ MIC $(\mu \mathrm{g} / \mathrm{mL})$} \\
\hline & ETP $(+\mathrm{CCCP})$ & MEM (+CCCP) & IMP (+CCCP) & ETP $(+\mathrm{PA} \beta \mathrm{N})$ & $\operatorname{MEM}(+\mathrm{PA} \beta N)$ & IMP (+PABN ) \\
\hline 12173 & $128(0.06)$ & $128(0.03)$ & $64(0.25)$ & $128(4)$ & $128(0.25)$ & $128(2)$ \\
\hline 13274 & $\square 256$ (0.25) & $128(0.03)$ & $64(0.25)$ & व256 (4) & $128(0.125)$ & $64(2)$ \\
\hline 131029 & $32(0.5)$ & $4(0.06)$ & $4(0.25)$ & $32(16)$ & $4(4)$ & $4(8)$ \\
\hline 12888 & $2(0.5)$ & $0.25(0.03)$ & $1(0.03)$ & $2(2)$ & $0.25(0.25)$ & $1(1)$ \\
\hline 13322 & $16(0.008)$ & $1(0.015)$ & $0.5(0.06)$ & $16(0.06)$ & $1(0.125)$ & $0.5(2)$ \\
\hline 15322 & $4(0.5)$ & $0.125(0.015)$ & $1(0.125)$ & $4(4)$ & $0.125(0.5)$ & 1 (1) \\
\hline 33127 & $32(16)$ & $2(1)$ & $2(1)$ & $32(64)$ & $2(4)$ & $2(4)$ \\
\hline 34102 & $4(1)$ & $0.25(0.06)$ & $1(0.5)$ & $4(2)$ & $0.25(0.25)$ & 1 (1) \\
\hline 15390 & $16(0.03)$ & $4(0.008)$ & $1(0.03)$ & $16(0.25)$ & $4(0.125)$ & $16(1)$ \\
\hline 11914 & $2(0.008)$ & $0.125(0.015)$ & $0.5(0.125)$ & $2(2)$ & $0.125(0.03)$ & $0.5(4)$ \\
\hline 13469 & $8(0.25)$ & $2(0.03)$ & $8(0.25)$ & $8(4)$ & $2(0.25)$ & $8(1)$ \\
\hline 16010 & $8(1)$ & $0.5(0.06)$ & $2(0.015)$ & $8(8)$ & $0.5(1)$ & $2(2)$ \\
\hline 13865 & $0.25(0.25)$ & $0.125(0.03)$ & $4(0.25)$ & $0.25(2)$ & $0.125(0.25)$ & 4 (1) \\
\hline
\end{tabular}

ETP, Etapenem; MEM, Meropenem; IPM, Imipenem; PAßN: Phe-Arg $\beta$-naphthylamide dihydrochloride; CCCP: carbonyl cyanide mchlorophenylhydrazone.

\section{Figures}

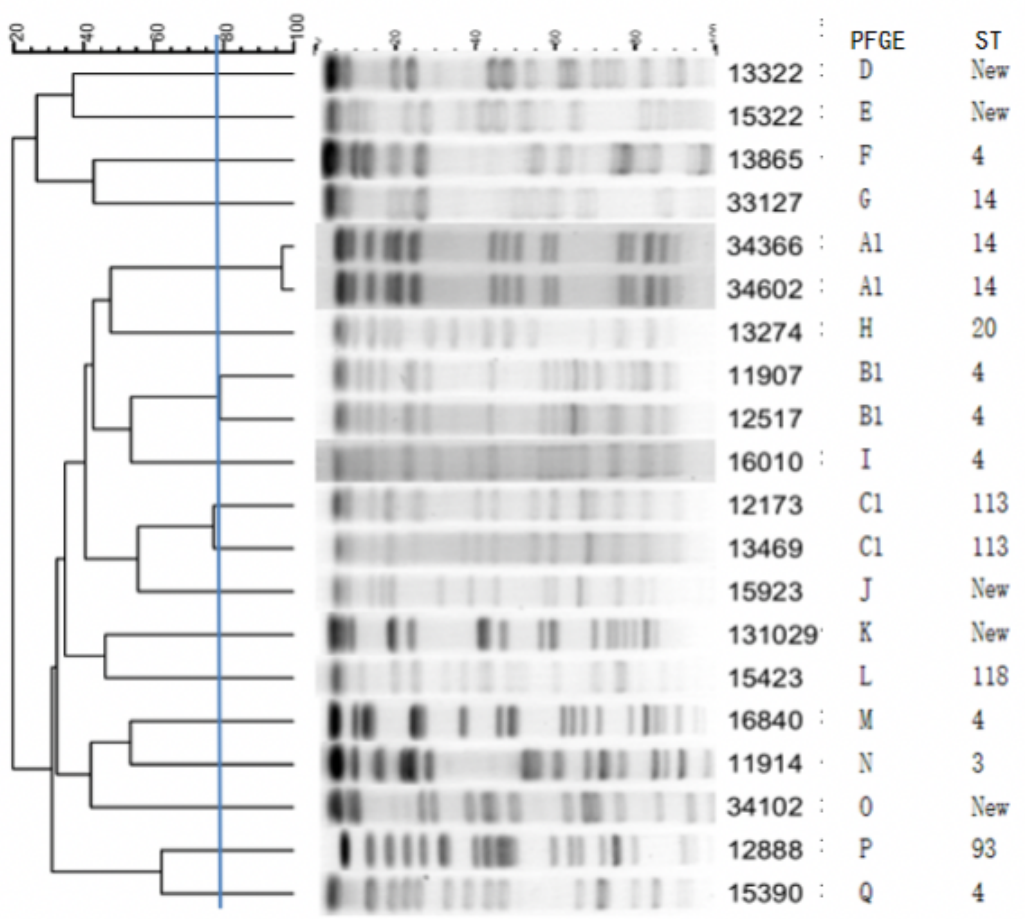

\section{Figure 1}

PFGE of Xbal-digested DNA and MLST 
(Fig. 2A)

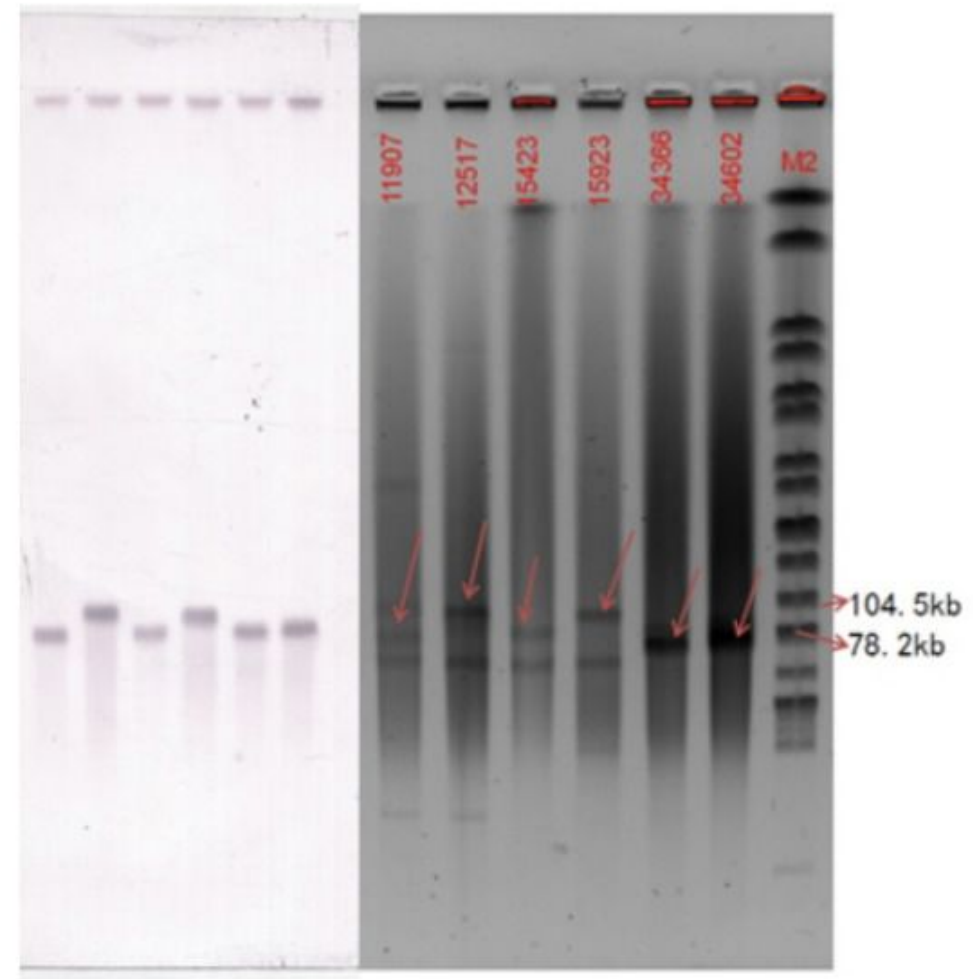

(Fig. 2B)

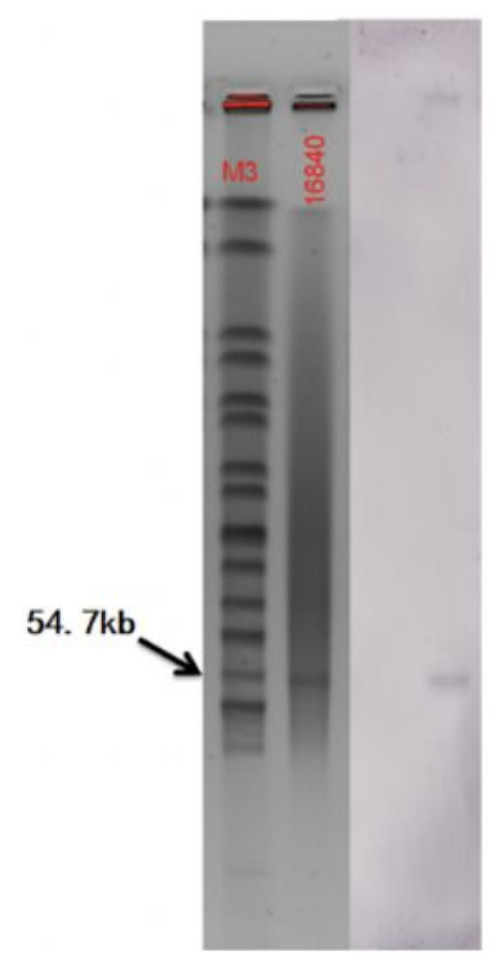

\section{Figure 2}

S1-digested plasmid DNA and Southern blot hybridization with blaKPC-2 (A) and blaNDM-1 (B).

\section{Supplementary Files}

This is a list of supplementary files associated with this preprint. Click to download.

- Supplmentalmaterials.docx 